\title{
MEASUREMENT OF THREE-DIMENSIONAL MAGNETOSTRICTION ON GRAIN-ORIENTED ELECTRICAL STEEL SHEET
}

\author{
Daisuke Wakabayashi — Takashi Todaka - Masato Enokizono *
}

\begin{abstract}
This paper deals with the magnetostriction of a grain-oriented electrical steel sheet measured by using the two-dimensional vector magnetic property measurement system and a three-axial strain gage. The magnetostrictions in arbitrary direction are calculated with the measured strain tensors. The magnetostriction characteristics were changed depending on the magnetization process according to the exciting magnetic flux density and the exciting direction. In this paper, we make clear the relationship among the two-dimensional magnetostriction, the magnetic flux density vector, and the magnetic field intensity vector in the grain- oriented electrical steel sheet.
\end{abstract}

K e y o r ds: vector magnetic property, three-dimensional magnetostriction, grain-oriented electrical steel sheet, magnetization process

\section{INTRODUCTION}

In recent years, development of low acoustic noise and high efficiency transformers are a very important subject. It is therefore necessary to precisely evaluate magnetic property and magnetostriction of grain-oriented electrical steel sheets. In the conventional measurements, the magnetic property and the magnetostriction are usually measured in the exciting direction. However, the magnetic property is a vector quantity and the magnetostriction occurs not only in the exciting direction but also in any direction. We can consider that the magnetostriction also occurs in thickness direction as shown in Fig. 1. This figure shows schematic view of the general threedimensional magnetostriction. The principal strain direction of the three-dimensional magnetostriction is not par- allel to the magnetic flux density vector $\boldsymbol{B}$ or the magnetic field intensity vector $\boldsymbol{H}[1,2]$.

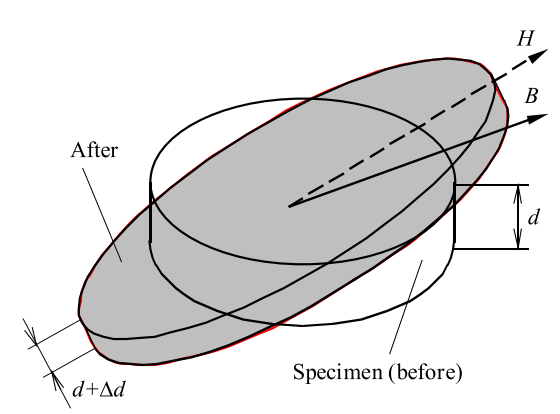

Fig. 1. Relationship among the three-dimensional magnetostriction and the vector $\boldsymbol{B}$, the vector $\boldsymbol{H}$

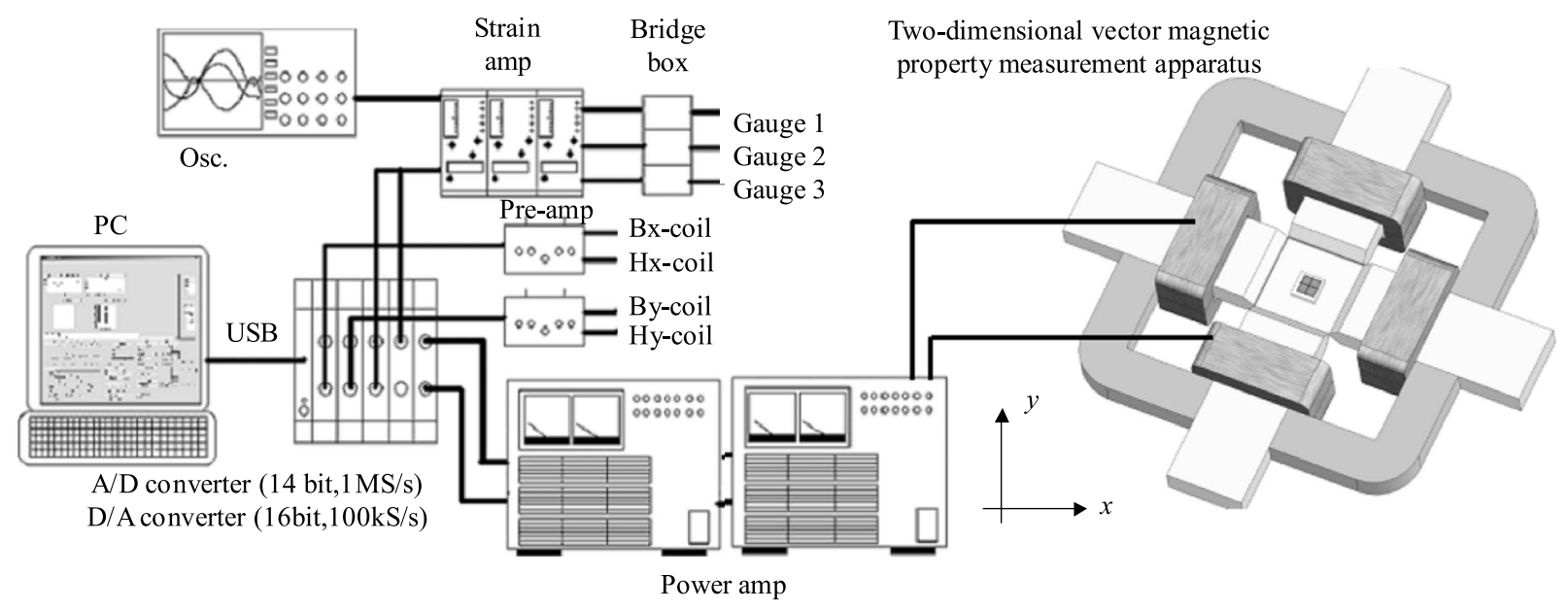

Fig. 2. Measurement system of the magnetostriction

\footnotetext{
* Department of Electrical and Electronic Engineering, Faculty of Engineering, Oita University, 700 Dannoharu Oita-shi, 870-1192, Japan, v10f1006@mail.cc.oita-u.ac.jp
} 


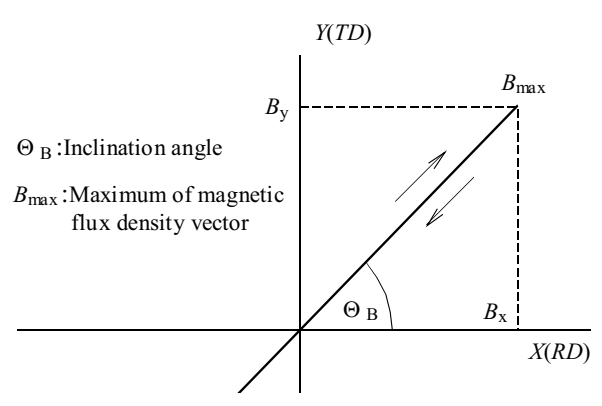

Fig. 3. Definition of the magnetic flux density condition

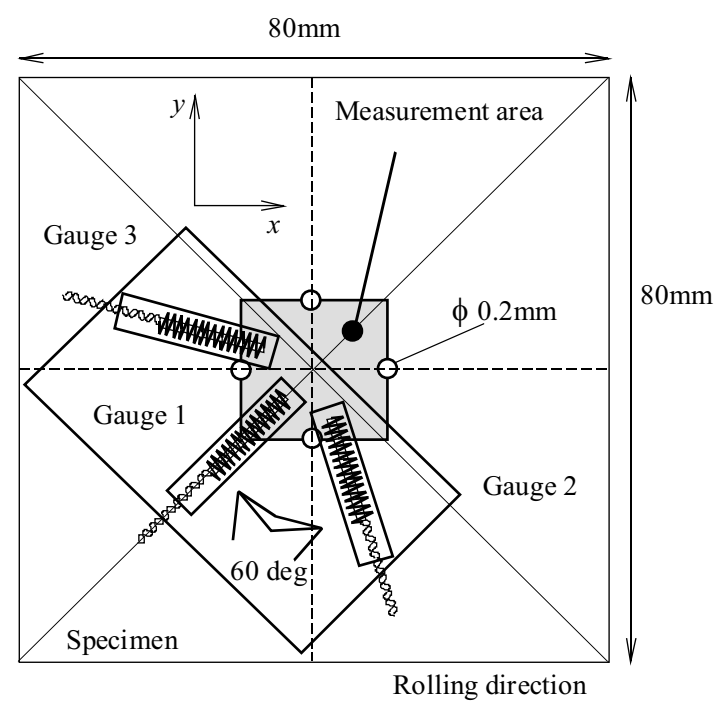

Fig. 4. Specimen and three-axial strain gauge

Table 1. The specification of the specimen

\begin{tabular}{lc}
\hline & $\begin{array}{c}\text { Grain-oriented } \\
\text { electrical steel sheet }\end{array}$ \\
\hline Grade & $35 \mathrm{P} 135$ \\
Size & $80 \mathrm{~mm} \times 80 \mathrm{~mm}$ \\
Thickness & $0.35 \mathrm{~mm}$ \\
Length of B-coil & $20 \mathrm{~mm}$ \\
number of turns of B-coil & $3 \mathrm{turn}$ \\
\hline
\end{tabular}

Table 2. Measurement conditions

\begin{tabular}{cccc}
\hline Specimen & $B_{\max }(\mathrm{T})$ & $\theta_{B}($ degree $)$ & $f(\mathrm{~Hz})$ \\
\hline $35 \mathrm{P} 135$ & $0.1-1.1$ & $0-90$ & 50 \\
\hline
\end{tabular}

We have measured the three-dimensional magnetostriction depending on the maximum magnetic flux density $B_{\max }$ and its inclination angle from the rolling direction $\theta_{B}$ by using the two-dimensional vector magnetic property measurement system and three-axial strain gage. In this paper, we make clear the relationship among the magnetic flux density vector $\boldsymbol{B}$ and the magnetic field strength vector $\boldsymbol{H}$ and the three-dimensional magnetostriction of a grain-oriented electrical steel sheet.

\section{MEASUREMENT SYSTEM}

\subsection{Measurement system for magnetostriction}

Figure 2 shows the measurement system of the magnetostriction based on the two-dimensional vector magnetic property measurement system $[3,4]$. The components of the magnetic flux density, $B_{x}$ and $B_{y}$ were measured with cross-type search coils (B-coils) wound through holes. The hole's diameter was about $0.2 \mathrm{~mm}$. The width of search coils was about $20 \mathrm{~mm}$. The components of the magnetic field strength, $H_{x}$ and $H_{y}$ were measured with a double $H$-coil. The $H_{x}$-coil was wound over a $16 \mathrm{~mm} \times 16 \mathrm{~mm} \times 1 \mathrm{~mm}$ ceramic plate and the $H_{y}$-coil was wound over the $H_{x}$-coil. The double $H$-coil was placed under the specimen and pressed to touch the specimen.

The criterion of measurement was the magnetic flux density waveform in each direction. The each pickup voltage form the B-coils was freed-back controlled to be sinusoidal to generate arbitrary alternating magnetic flux in the measuring area, such as an alternating magnetic field inclined any direction. The convergence was judged with the amplitude, the phase, and the distortion factor of the pickup voltages. After the convergence, we adjusted the strain amplifier (KYOWA DPM-611) and measured the magnetostriction with a strain gage. Random noises were reduced by averaging the 32 cycles waveforms.

Figure 3 shows the alternating magnetic flux density condition. This condition is expressed with two parameters. They are the maximum magnetic flux density $B_{\max }$ and the inclination angle $\theta_{B} . \theta_{B}$ is an angle between the direction of $B_{\max }$ and the rolling direction. The rolling direction was assumed to be 0 deg.

\subsection{Specimen and three-axial strain gauge}

Figure 4 shows the specimen and the three-axial strain gauge. Table 1 shows the specification of the specimen. The three-axial strain gauge (KYOWA SKF-5964) is a non-induction gauge. It is necessary to measure strains in three directions to derive the strain tensor and to estimate magnetostriction in arbitrary direction.

\subsection{Two-dimensional magnetostriction}

In order to obtain the magnetostriction in arbitrary direction, we used the following expression. Usually, the magnetostriction can be expressed as

$$
\lambda=\frac{L}{\Delta L}=\sum_{i=1}^{2} \sum_{j=1}^{2} e_{i j} \beta_{i} \beta_{j}=e_{11} \beta_{1} \beta_{1}+2 e_{12} \beta_{1} \beta_{2}+e_{22} \beta_{2} \beta_{2}
$$




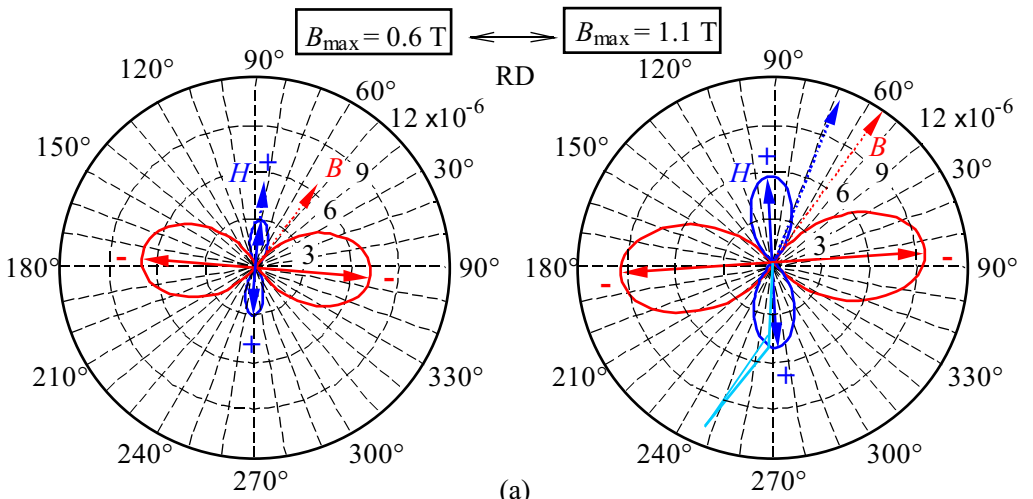

(a)

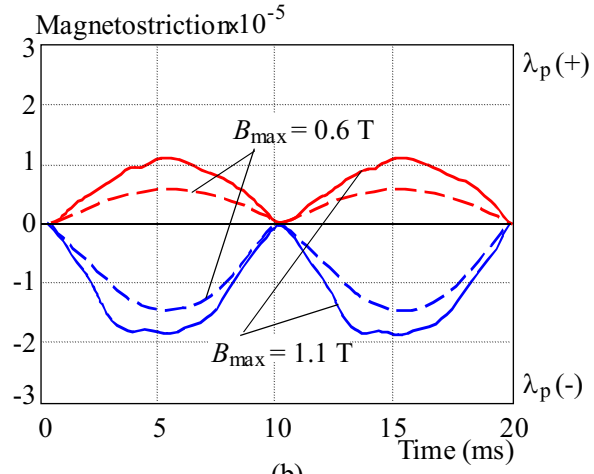

(b)

Fig. 5. Evaluation of the two-dimensional magnetostriction: (a) - two-dimensional magnetostriction, (b) - principal strain waveforms $\left(\theta_{B}=55 \mathrm{deg}\right)$

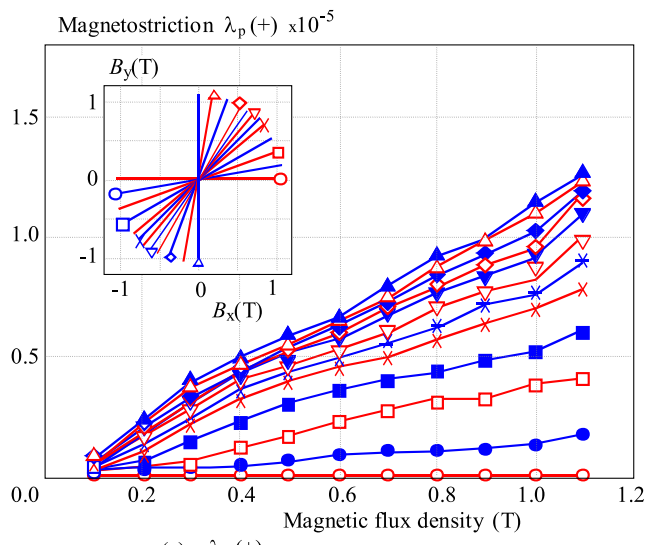

(a) $\lambda_{\mathrm{p}}(+)$

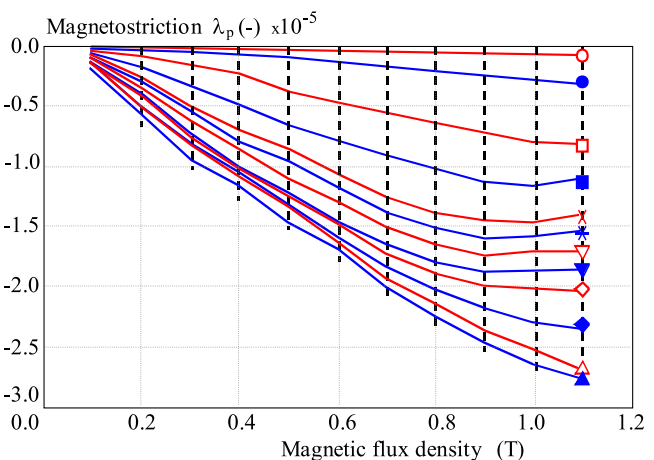

(b) $\lambda_{\mathrm{p}}(-)$

Fig. 6. $\lambda_{p}(+)$ and $\lambda_{p}(-)$ depending on $B_{\max }$ and $\theta_{B}$

where, $\lambda$ is the magnetostriction and $e_{i j}$ is the strain tensor. $\beta_{i}$ or $\beta_{j}$ is the component of the unit vector. This equation can be rewritten as follows,

$$
\left(\begin{array}{l}
e_{11} \\
e_{12} \\
e_{22}
\end{array}\right)=\left[\begin{array}{ccc}
(1-\sqrt{3}) / 3 & 1 / 3 & (1+\sqrt{3}) / 3 \\
1 / 3 & -2 / 3 & 1 / 3 \\
(1+\sqrt{3}) / 3 & 1 / 3 & (1-\sqrt{3}) / 3
\end{array}\right]\left(\begin{array}{c}
\lambda_{\text {gauge2 }} \\
\lambda_{\text {gauge }} \\
\lambda_{\text {gauge3 }}
\end{array}\right)
$$

By substituting the strain tensor calculated with the equation (2) into the equation (1), we can obtain the magnetostrictions in arbitrary direction. We call this the two-dimensional magnetostriction.

\section{EXPERIMENTAL RESULTS AND DISCUSSIONS}

\subsection{Measurement conditions}

Table 2 shows the measurement conditions. $B_{\max }$ was changed from $0.1 \mathrm{~T}$ to $1.1 \mathrm{~T}$ per $0.1 \mathrm{~T}$ step and $\theta_{B}$ was changed from 0 deg to $90 \mathrm{deg}$. The excitation frequency was $50 \mathrm{~Hz}$.

\subsection{Evaluation of two-dimensional magnetostric- tion}

Figure 5 shows the measured results of the twodimensional magnetostriction. The grey line shows the expansion and the grey arrow shows the principal strain of the expansion, $\lambda_{p}(+)$, which is the maximum magnetostriction. The black line shows the contraction and the black arrow shows the principal strain of the contraction, $\lambda_{p}(-) . \lambda_{p}(+)$ occurred in $90 \mathrm{deg}$. and $\lambda_{p}(-)$ occurred in 0 deg. The principal strains, $\boldsymbol{B}$, and $\boldsymbol{H}$ were not parallel. We can point out that the conventional measurement is not enough. Figure 5(b) shows the $\lambda_{p}(+)$ and $\lambda_{p}(-)$ waveforms.

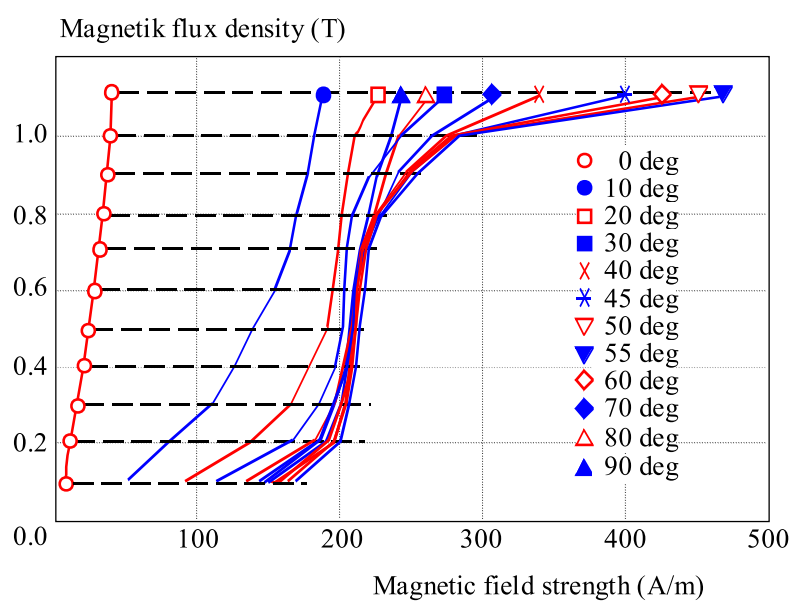

Fig. 7. $\lambda_{p}(-)$ waveforms depending on $B_{\max }$ at $\theta_{B}=55 \mathrm{deg}$ 


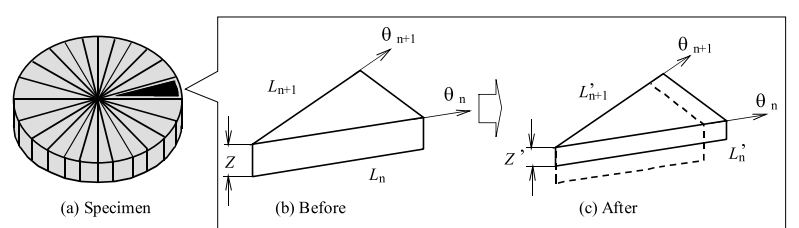

Fig. 8. $\lambda_{p}(-)$ waveforms depending on $\theta_{B}$ at $B_{\max }=1.1 \mathrm{~T}$

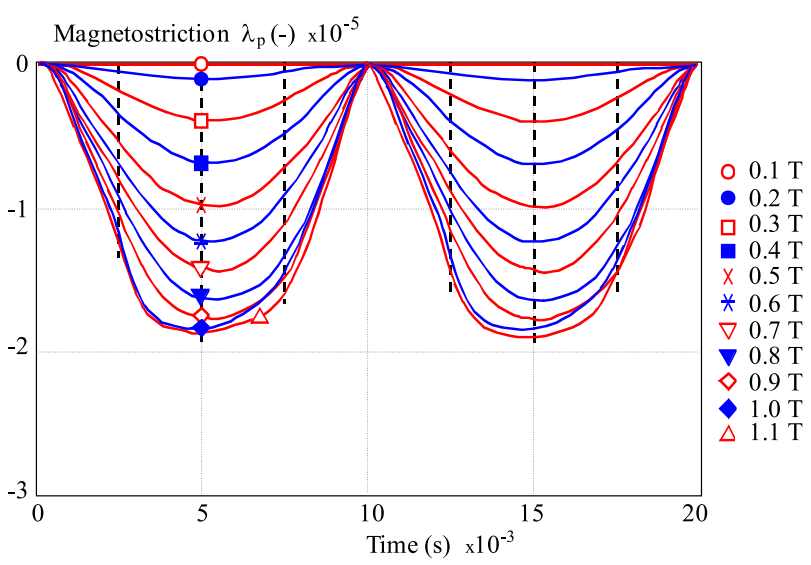

Fig. 9. B-H curves

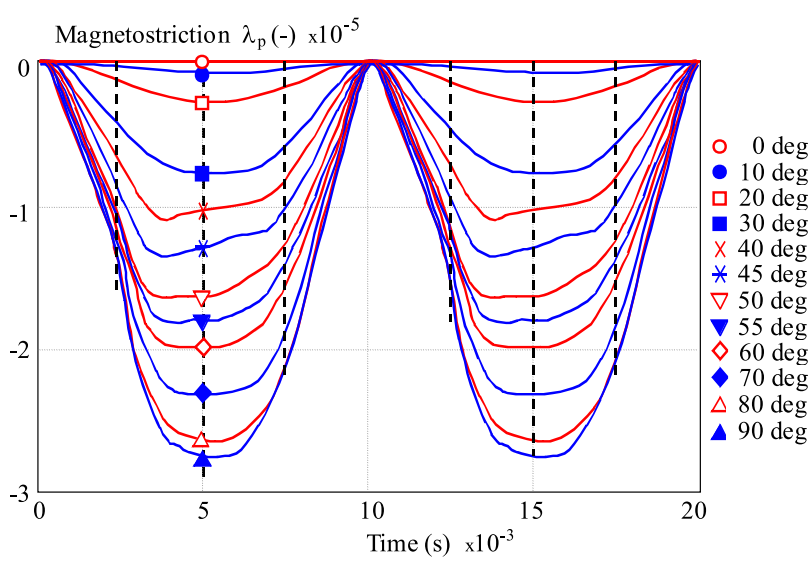

Fig. 10. Model for calculating $\lambda_{z}$

\subsection{Magnetostriction depending on $B_{\max }$ and $\theta_{B}$}

Figure 6 shows $\lambda_{p}(+)$ and $\lambda_{p}(-)$ depending on $B_{\max }$ and $\theta_{B} \cdot \lambda_{p}(+)$ and $\lambda_{p}(-)$ increased with increasing $B_{\max }$ and $\theta_{B}$. The magnetostriction became the minimum at $\theta_{B}=0$ deg. (the rolling direction) and the maximum at $\theta_{B}=90 \mathrm{deg}$. (the transverse direction). This result is well known in the conventional measurement and called the magnetostriction anisotropy [5]. However, as shown in Fig. 6(b), the $\lambda_{p}(-)$ decreased with increasing $B_{\max }$ near $\theta_{B}=55 \mathrm{deg}$. Figure 7 shows $\lambda_{p}(-)$ waveforms depending on $B_{\max }$ at $\theta_{B}=55 \mathrm{deg}$. as an example. At the higher magnetic flux condition, the $\lambda_{p}(-)$ waveforms were distorted and peak-to-peak $\lambda_{p}(-)$ did not increase due to the distortions.

Figure 8 shows $\lambda_{p}(-)$ waveforms depending on $\theta_{B}$ at $B_{\max }=1.1 \mathrm{~T}$. As shown in this figure, the distortions of $\lambda_{p}(-)$ waveforms were changed depending on $\theta_{B}$. This phenomenon can be explained with the $\mathrm{B}-\mathrm{H}$ curves as shown in Fig. 9. In this figure, the magnetic field strength corresponds to the maximum field strength $H_{\max }$ and the magnetic flux density is the maximum flux density $B_{\max }$. At $B_{\max }$ was $0.6 \mathrm{~T}, H_{\max }$ in $\theta_{B}=90 \mathrm{deg}$ was larger than one in $\theta_{B}=55 \mathrm{deg}$. Inversely, at $B_{\max }$ was $1.1 \mathrm{~T}, H_{\max }$ in $\theta_{B}=55 \mathrm{deg}$ was larger than one in $\theta_{B}=90 \mathrm{deg}$. It is evident that the different magnetization processes depending on $\theta_{B}$ influenced the magnetostriction.

The magnetostriction was larger in $\theta_{B}=90 \mathrm{deg}$ in comparison with that in $\theta_{B}=55 \mathrm{deg}$, because a hard magnetizing direction around $\theta_{B}=55$ deg existed due to crystal anisotropy at the high magnetic field condition and in this state the rotational magnetization process should be main. As a result, it can be considered that the magnetostriction waveforms are distorted near $\theta_{B}=$ $55 \mathrm{deg}$. We can point out that it is important to measure the magnetic field strength with the magnetostriction to know the magnetization state.

\subsection{Magnetostriction in thickness direction}

The magnetostriction in thickness direction, $\lambda_{z}$ is calculated with the measured data of the two-dimensional magnetostriction. Figure 10 shows the model for calculating $\lambda_{z}$. The specimen is divided into elements every direction. We can consider that the triangular pole element changes as shown in Figs. 10(b) and (c). Therefore, under assumption of constant volume, $\lambda_{z}$ can be written as follows,

$$
\lambda_{z}=\frac{1}{2} \frac{1}{\left(1+\lambda_{\theta n}\right)\left(1+\lambda_{\theta n+1}\right)}-1
$$

where, $\lambda_{\theta n}$ is the magnetostriction in $\theta_{n}$ direction. We can estimate the three-dimensional magnetostriction including $\lambda_{z}$.

Figure 11 shows an example of the three-dimensional magnetostriction. Figure 11(a) shows the modified shape of the specimen due to three-dimensional magnetostriction. The thickness in the rolling direction increased and one in the transverse direction decreased. Figure 11(b) shows the magnetostriction waveforms in thickness direction at point $\mathrm{A}$ and $\mathrm{B}$ defined in Fig. 11(a). $\lambda_{z}$ at the point $\mathrm{A}$ was larger than one at the point $\mathrm{B}$. The magnetostriction in thickness direction also occurs similar to the magnetostriction in plane direction.

\section{CONCLUSIONS}

The three-dimensional magnetostriction property of the grain-oriented electrical steel sheet depending on the 
maximum magnetic flux density $B_{\max }$ and the inclination angle $\theta_{B}$ are measured by using the two-dimensional vector magnetic property measurement system and threeaxial strain gage. The principal strain of the expansion occurred in $90 \mathrm{deg}$ and one of the contraction occurred in 0 deg. without depending on the inclination angle $\theta_{B}$. They were not parallel to the magnetic flux density vector, $\boldsymbol{B}$ and the field strength vector, $\boldsymbol{H} . \lambda_{p}(+)$ and $\lambda_{p}(-)$ increased with increasing $B_{\max }$ and $\theta_{B}$. However, the $\lambda_{p}(-)$ decreased with increasing $B_{\max }$ near $\theta_{B}=55 \mathrm{deg}$. The hard magnetizing direction around $\theta_{B}=55$ deg existed due to crystal anisotropy at the high magnetic field condition and the rotational magnetization process is main in this state, therefore the magnetostriction was larger in $\theta_{B}=90 \mathrm{deg}$ in comparison with that in $\theta_{B}=55 \mathrm{deg}$. From the above reason, the magnetostriction waveforms were distorted near $\theta_{B}=55 \mathrm{deg}$.
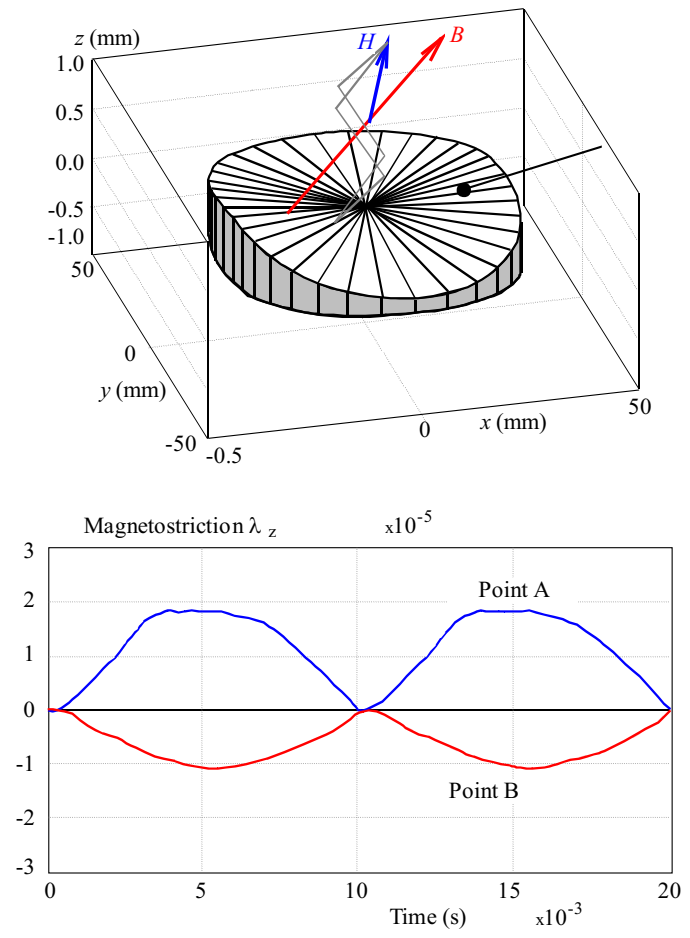

Fig. 11. Three-dimensional magnetostriction at $B_{\max }=1.1 \mathrm{~T}$, $\theta_{B}=55 \mathrm{deg}$

In this paper, we have pointed out that it is important to measure the magnetic field strength with the magnetostriction to know the magnetization state in the same time and the magnetostriction in thickness direction to evaluate three-dimensional magnetostrictions in detail.

\section{REFERENCES}

[1] WAKABAYASHI, D.-MAEDA, Y.-SHIMOJI, H.-TODAKA, T.-ENOKIZONO, M.: Measurement of Vector Magnetostriction in Alternating and Rotating Magnetic Field, Przeglad Elektrotechniczny 85 No. 1 (2009), 34-38.

[2] WAKABAYASHI, D.-TAKASHI, T.-ENOKIZONO, M. : Three-Dimensional Magnetostriction and Vector Magnetic Properties under Alternating Magnetic Flux Conditions in Arbitrary Direction, IEEJ Transactions on Fundamentals and Materials 130 No. 4 (2010), 387-393.

[3] EnOKizOnO, M.: Two-Dimensional Vector Magnetic Property, Journal of the Magnetics Society of of Japan 27 No. 2 (2003), 50-58.

[4] EnOKizono, M.-SIEverT, J. D.: Numerical Analysis of Accuracy of Rotational Magnetic Loss Measurement Apparatus, IEEE Tranlation Journal of Magnetics in 5 No. 9 (Sep 1990), $742-748$.

[5] SOMKUN, S.-MOSES, A. J.-ANDERSON, P. I.-KLIMCZYK, P.: Magnetostriction Anisotropy and Rotational Magnetostriction of a Nonoriented Electrical Steel, IEEE Transactions on Magnetics 46 No. 2 (Feb 2010), 302-305.

Received 30 September 2010

Daisuke Wakabayashi was born in 1985 . He received the MEng degrees in electrical engineering from Oita University, Japan in 2010, respectively. He is currently a doctor's course student at Oita University, Japan.

Takashi Todaka was born in 1960. He is currently an Associate Professor at Department of Electrical and Electronic Engineering, Faculty of Engineering at Oita University, Japan. His main research area is in magnetic engineering. His recent research is development of ferro-magnetic shape memory alloy and its applications.

Masato Enokizono was born in 1949. He is currently a Professor at Department of Electrical and Electronic Engineering, Faculty of Engineering at Oita University, Japan. He got research scholarship of Alexander von Humboldt-Stifung in Phusikalisch-Technischen Bundesanstalt(PTB) Braunschweig, Lab.

This work was presented at International Conference Magnetic Measurement 2010, Prague, 12-15 September 2010, Czech Republic. 